EPJ manuscript No.

(will be inserted by the editor)

\title{
Measuring effective temperatures in out-of-equilibrium driven systems
}

\author{
Raphaël Exartier ${ }^{1,2, a}$ and Luca Peliti ${ }^{2,3, b, c}$ \\ 1 Laboratoire des Milieux Désordonnés et Hétérogènes ${ }^{\mathrm{d}}$ \\ Tour 13 - Case 86, 4 place Jussieu, F-75252 Paris Cedex 05 (France) \\ 2 Dipartimento di Scienze Fisiche and Unità INFM, Università "Federico II" \\ Mostra d'Oltremare, Pad. 19, I-80125 Napoli (Italy) \\ 3 Laboratoire de Physico-Chimie Théorique \\ Ecole Supérieure de Physique et Chimie Industrielles \\ 10, rue Vauquelin, F-75231 Paris Cedex 05 (France)
}

Received: date / Revised version: date

\begin{abstract}
We introduce and solve a model of a thermometric measurement on a driven glassy system in a stationary state. We show that a thermometer with a sufficiently slow response measures a temperature higher than that of the environment, but that the measured temperature does not usually coincide with the effective temperature related to the violation of the Fluctuation-Dissipation Theorem.
\end{abstract}

PACS. 05.70.Ln Nonequilibrium thermodynamics, irreversible processes - 07.20.Dt Thermometry 61.43.Fs Glasses - 75.10.Nr Spin-glass and other random models

\section{Introduction}

Thermal equilibrium is a rather subtle concept. It relies on the distinction between "fast" and "slow" processes with respect to a given macroscopic measurement. It follows that the same system can be at equilibrium on one scale and out of equilibrium on another. More strikingly it can be at equilibrium but exhibiting different properties on two scales at once [1].

The notion most intimately connected to equilibrium is temperature. It is operationally defined by the so-called zeroth law of thermodynamics, which states that when two systems are in thermal equilibrium with a third one, then they must be in thermal equilibrium with each other. This allows one to define temperature as a signature of the equivalence class defined by mutual thermal equilibrium. This property makes possible the use of test systems, called "thermometers", to decide whether any two systems will or will not remain in thermal equilibrium when brought into contact. When two systems are not in mutual equilibrium, the direction of the energy flow between them is determined by the second law of thermodynamics.

${ }^{a}$ E-mail: exartier@ccr.jussieu.fr

b E-mail: peliti@na.infn.it

c Associato INFN, Sezione di Napoli (Italy).

d Laboratoire associé au CNRS (URA nº 800) et à l'Université Pierre-et-Marie Curie-Paris VI.

Correspondence to: Raphaël Exartier.
When dealing with non-equilibrium systems the challenge is thus to produce an "effective" time-scale dependent temperature that would predict the direction of heat flows within this scale.

Indeed, in the context of weak turbulence, Hohenberg and Shraiman [2] have defined an effective temperature for stationary non-equilibrium systems through an expression involving the response, the correlation and the temperature of the heat reservoir. A closely related expression appears in the theory of aging systems [3]. In a recent work 佃, these views have been unified for a class of out-ofequilibrium systems with small heat flows, which includes nonstationary pure relaxational systems, like glasses, and stationary systems, slowly driven by non-relaxational forces. The concept of effective temperatures has been further reviewed in refs. [5, 66. In a recent experiment temperatures higher than the thermal bath temperatures have been exhibited in an oscillating circuit coupled to an aging glycerol sample after a quench [7].

In the present work we analyze the process of a thermometric measurement in a glassy system, by means of an exactly solvable model. We restrict ourselves to the stationary non-equilibrium regime of a driven system. We consider a simple system and a thermometer, both described by Langevin equations. By taking advantage of Time-Translation Invariance (TTI) the Langevin equation is transformed into an algebraic equation in Fourier space, that can be analytically solved. 
Raphaël Exartier, Luca Peliti: Measuring effective temperatures

Our thermometer is a simple physical system coupled to its own heat bath, which is different from the thermal bath of the observed system. We suppose that during the measuring time, the two systems are brought into contact, each being coupled with its own thermal bath. We then monitor the exchanged energy between the system and the thermometer in the stationary regime. The reading of the thermometer corresponds to the temperature of the heat bath of the thermometer for which the net energy flow between the system and the thermometer vanishes. We discuss the relation of the measured temperature with the effective temperature defined in refs. [2, 4 .

In section 2 we recall the generalization of the Fluctuation-Dissipation Theorem to nonequilibrium systems and show how it defines an effective temperature. In section 3 we describe the general measurement procedure. In section 4 we specify the procedure for the measurement of the effective temperature of an asymmetric spherical SK model. Finally, section 5 is devoted to the analysis of the results obtained for this system.

\section{Fluctuation-Dissipation Theorem and effective temperatures}

According to [4], the definition of an effective temperature for non-equilibrium systems can be related to the violation of the Fluctuation-Dissipation Theorem (FDT). Let us consider a system (described by the Hamiltonian $H$ ) subject to a time-dependent perturbation of the form:

$$
H \longrightarrow H-h(t) O
$$

where $O$ is an extensive operator. The correlation function $C\left(t, t^{\prime}\right)$ of $O$ is defined by

$$
\begin{aligned}
C\left(t, t^{\prime}\right) & :=\langle O(t) O(t-)\rangle_{\mathrm{c}} \\
& :=\left\langle O(t) O\left(t^{\prime}\right)\right\rangle-\langle O(t)\rangle\left\langle O\left(t^{\prime}\right)\right\rangle,
\end{aligned}
$$

while the corresponding response function $R\left(t, t^{\prime}\right)$ is given by

$$
R\left(t, t^{\prime}\right):=\left.\frac{\delta\langle O(t)\rangle}{\delta h\left(t^{\prime}\right)}\right|_{h \equiv 0} .
$$

For a system at equilibrium with a thermal reservoir at temperature $T$, Time-Translation Invariance (TTI) intimates that both the correlation and the response functions depend only on the time difference $\tau$ between their time arguments $\left(\tau=t-t^{\prime}\right)$. On the other hand, the FDT entails a relation between the response and the correlation functions:

$$
R(\tau)=\frac{\theta\left(t-t^{\prime}\right)}{T} \frac{\partial}{\partial t^{\prime}}\left\langle O(t) O\left(t^{\prime}\right)\right\rangle_{\mathrm{c}}=-\frac{\theta(\tau)}{T} \frac{\partial C(\tau)}{\partial \tau} .
$$

Experimentally, one usually measures the (time-)integrated susceptibility:

$$
\chi(\tau):=\int_{0}^{\tau} d \tau^{\prime} R\left(\tau^{\prime}\right) .
$$

At equilibrium, we can use the FDT to compare this susceptibility to the correlation function:

$$
\chi(\tau)=\frac{1-C(\tau)}{T}
$$

(We are considering magnetic systems for which $C(0)=$ 1.) Then, a parametric plot of $\chi(\tau)$ vs. $C(\tau)$ yields a straight line with slope equal to $-1 / T$.

A certain class of out of equilibrium systems with very slow dynamics exhibits an aging regime in which the FDT is violated in a very specific way. Following an initial quench of temperature, these systems fall out of equilibrium and do not reach it again, even on macroscopic time scales. The longer the time $t_{\mathrm{w}}$ elapsed since the initial quench, the slower is the response of the system to a given perturbation: the system ages. This phenomenon also appears in the time-correlation functions. In these systems, even in the limit $t_{\mathrm{w}} \rightarrow \infty$, a parametric plot of $\chi\left(t, t_{\mathrm{w}}\right) v s$. $C\left(t, t_{\mathrm{w}}\right)$ does not yield a straight line with slope $-1 / T$ as in equilibrium.

Very similar features appear in some stationary nonequilibrium (driven) systems [3, 4, 5, 6, 8], like the one shown in figure 1. Let us introduce a parameter $v$ which measures

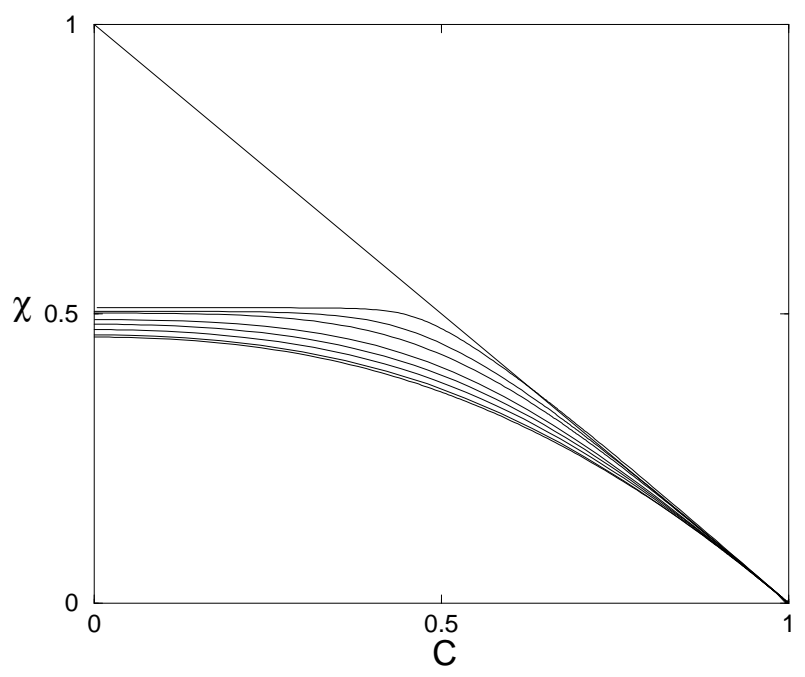

Fig. 1. Plot of $\chi v s$. C of the asymmetric spherical SK model for $T_{1}=10, J=20$ and different values of the asymmetry parameter $v$. The susceptibility $\chi$ is normalized by the bath temperature $T_{1}$. The lines correspond (from above to below) to $v=0.2 ; 0.3 ; 0.4 ; 0.5 ; 0.6 ; 0.7 ; 0.8$.

the intensity of the driving force. (For these systems, of course, the time needed to reach the stationary nonequilibrium state diverges as $v \rightarrow 0$.) In a sense, $v$ plays a role similar to $t_{\mathrm{w}}$ in aging systems: the smaller $v$, the "older" the system. In a driven system TTI is satisfied, but FDT is not, even in the limit of vanishing driving force $(v \rightarrow 0)$ [4]. Let us consider the slope $\chi^{\prime}(C)=d \chi / d C$ of the curve $\chi(C)$. According to [4], for small enough driving forces, the effective temperature $T^{\mathrm{eff}}$ can be expressed in terms 
of this slope:

$$
T^{\mathrm{eff}}(C):=-\frac{1}{\chi^{\prime}(C)}=-\left(\frac{d \chi(C)}{d C}\right)^{-1} .
$$

In all known cases one has $T^{\mathrm{eff}}(C) \geq T$.

We can define a time scale $\tau(q, v)$ by means of the relation:

$$
C(\tau(q, v), v)=q .
$$

If $q$ is larger than a threshold value $q_{\mathrm{EA}}$ (called the Edwards-Anderson order parameter) one has

$$
\lim _{v \rightarrow 0} \tau(q, v)<\infty
$$

This is equivalent to the following definition of $q_{\mathrm{EA}}$ :

$$
q_{\mathrm{EA}}:=\lim _{\tau \rightarrow \infty} \lim _{v \rightarrow 0} C(\tau, v) .
$$

On the other hand, if $q<q_{\mathrm{EA}}$, the time $\tau(v)$ diverges as $v$ goes to zero.

Let us thus consider a thermometric measurement, performed on a characteristic time scale $\tau$. We wish to compare it with $T^{\mathrm{eff}}(\tau)$, where

$$
T^{\mathrm{eff}}(\tau):=-\left.\frac{1}{\chi^{\prime}(q)}\right|_{q=C(\tau, v)} .
$$

\section{Measurement procedure}

The measurement procedure is similar to the one described

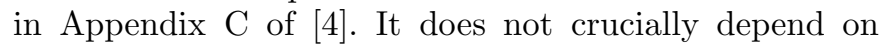
the nature of the thermometer, as long as it satisfies the fluctuation-dissipation theorem and has a tunable response time. We use a small but macroscopic thermometer in contact with a thermal bath at temperature $T_{2}$. The driven system whose effective temperature is to be measured is in contact with a bath at temperature $T_{1}$.

The thermometer is coupled to the observable $O_{1}\left(S_{1}\right)$ of the system via its observable $\mathrm{O}_{2}\left(S_{2}\right)$. The interaction Hamiltonian writes: $H_{\text {int }}=-a O_{1}\left(S_{1}\right) O_{2}\left(S_{2}\right)$. We remark that $O_{1}$ and $O_{2}$ are conjugate to each other. After a certain time, which depends on the coupling constant $a$ and on $\left|T_{1}-T_{2}\right|$, a stationary regime appears.

We define as $T^{\text {meas }}$ the value of the temperature $T_{2}$ for which the net energy transfer between the system and the thermometer vanishes at stationarity. This temperature is compared with the effective temperature $T^{\text {eff }}$ of the system, defined by eq. (7).

During the measurement procedure both the system and the thermometer are kept in contact with their own baths. The resulting system has two temperatures. (A simple system with two temperatures has been introduced and discussed in ref. [9].)

At stationarity, the net power gain for the thermometer, $\dot{Q}_{2}$, writes:

$$
\dot{Q}_{2}=a\left\langle\dot{O}_{1} O_{2}\right\rangle=\lim _{\tau \rightarrow 0} a \partial_{\tau} \tilde{C}_{12}(\tau),
$$

where we have introduced the cross correlation function

$$
\tilde{C}_{12}(\tau):=\lim _{t \rightarrow \infty}\left\langle O_{1}(t+\tau) O_{2}(t)\right\rangle_{\mathrm{c}} .
$$

Using linear response, one has

$$
\begin{aligned}
& O_{1}(t)=O_{1 \mathrm{~b}}(t)+a \int_{0}^{t} d t R_{1}\left(t-t^{\prime}\right) O_{2 \mathrm{~b}}\left(t^{\prime}\right), \\
& O_{2}(t)=O_{2 \mathrm{~b}}(t)+a \int_{0}^{t} d t R_{2}\left(t-t^{\prime}\right) O_{1 \mathrm{~b}}\left(t^{\prime}\right),
\end{aligned}
$$

where $O_{i \mathrm{~b}}$ are the observables in the absence of coupling and $R_{i}$ the corresponding response function $(i=1,2)$. At stationarity, to first order in the coupling $a$, the cross correlation function (12) is given by

$$
\begin{aligned}
\tilde{C}_{12}(\tau)= & a \int_{-\infty}^{\tau} d \tau^{\prime} R_{1}\left(\tau-\tau^{\prime}\right) C_{2}\left(\tau^{\prime}\right) \\
& +a \int_{-\infty}^{0} d \tau^{\prime} R_{2}\left(-\tau^{\prime}\right) C_{1}\left(\tau^{\prime}-\tau\right),
\end{aligned}
$$

where we have introduced the correlation functions of the bare systems:

$$
C_{i}(\tau):=\lim _{t \rightarrow \infty}\left\langle O_{i \mathrm{~b}}(t+\tau) O_{i \mathrm{~b}}(t)\right\rangle_{\mathrm{c}} .
$$

Then the rate of heat transfer writes:

$$
\dot{Q}_{2}=a^{2} \int_{0}^{\infty} d \tau\left(R_{2}(\tau) \partial_{\tau} C_{1}(\tau)-R_{1}(\tau) \partial_{\tau} C_{2}(\tau)\right) .
$$

From eq. (8), we can substitute $q$ for $\tau$ as the integration variable: $d q=\dot{C}_{1}(\tau) d \tau$. One can now exploit the fluctuation dissipation relations for the bare systems, namely $R_{1}(\tau)=-\dot{C}_{1}(\tau) / T^{\mathrm{eff}}(\tau)$ and $R_{2}(\tau)=-\dot{C}_{2}(\tau) / T_{2}$, and obtain

$$
\dot{Q}_{2}=a^{2} \int_{0}^{1} d q R_{2}(q)\left(\frac{T_{2}}{T^{\mathrm{eff}}(q)}-1\right) .
$$

The measured temperature $T^{\text {meas }}$ is defined as the one which makes $\dot{Q}_{2}$ to vanish:

$$
T^{\text {meas }}\left(q_{2}\right)^{-1}:=\frac{\int_{0}^{1} d q R_{2}\left(q, q_{2}\right) T^{\mathrm{eff}}(q)^{-1}}{\int_{0}^{1} d \tau R_{2}\left(q, q_{2}\right)} .
$$

We have introduced the parameter $q_{2}:=C_{1}\left(\tau_{2}\right)$, where $\tau_{2}$ represents the tunable characteristic time of the thermometer. $T^{\text {meas }}\left(q_{2}\right)^{-1}$ is the average of $T^{\mathrm{eff}}(q)^{-1}$ weighted by $R_{2}\left(q, q_{2}\right)$. We remark that the measured temperature is independent of the coupling constant $a$, provided that it is small enough to ensure the validity of the linear response theory. On the other hand, $T^{\text {meas }}$ depends strongly on $q_{2}$, because the lower boundary of the integrals appearing in eq. (19) is effectively cutoff at $q_{2}$.

For a system at equilibrium $T^{\mathrm{eff}}(q)=T_{1}$ for any $q$. Thus $T^{\text {meas }}=T_{1}$ as expected, whatever the characteristic time of the thermometer. 
Let us consider a simple system with only two time sectors:

$$
T^{\mathrm{eff}}(\tau)= \begin{cases}T_{1}, & \text { for } \tau \leq \tau_{\mathrm{EA}} \\ T_{1}^{\prime}>T_{1}, & \text { for } \tau \geq \tau_{\mathrm{EA}}\end{cases}
$$

We have introduced the notation $\tau_{\mathrm{EA}}$ defined by the relation: $C_{1}\left(\tau_{\mathrm{EA}}\right)=q_{\mathrm{EA}}$. For $q_{2} \geq q_{\mathrm{EA}}$, which corresponds to probing the short time behaviour of the aging system, the lower boundary cutoff at $q_{2}$ of the integrals of eq. (19) implies that the effective temperature of the driven system is constantly $T^{\mathrm{eff}}(q)=T_{1}$ over the integration interval and thus:

$$
T^{\text {meas }}\left(q_{2}\right)=T_{1} .
$$

For $q_{2} \leq q_{\mathrm{EA}}$ the temperature $T^{\text {meas }}$ is not equal to $T^{\mathrm{eff}}=$ $T_{1}^{\prime}$. Splitting the numerator of $(19)$ in two integrals from 0 to $q_{\mathrm{EA}}$ and from $q_{\mathrm{EA}}$ to 1 we obtain

$$
\begin{aligned}
T^{\text {meas }}\left(q_{2}\right)^{-1}= & \frac{1}{T_{1}^{\prime}} \frac{\int_{0}^{q_{\mathrm{EA}}} d q R_{2}\left(q, q_{2}\right)}{\int_{0}^{1} d \tau R_{2}\left(q, q_{2}\right)} \\
& +\frac{1}{T_{1}} \frac{\int_{q_{\mathrm{EA}}}^{1} d q R_{2}\left(q, q_{2}\right)}{\int_{0}^{1} d \tau R_{2}\left(q, q_{2}\right)} .
\end{aligned}
$$

The measured inverse temperature is a weighted average of the inverse temperature of the bath $1 / T_{1}$ and the inverse effective temperature $1 / T^{\mathrm{eff}}$. Its value is intermediate between them, as shown in figure 2, where the aging system and the thermometer are the ones described in the next section.

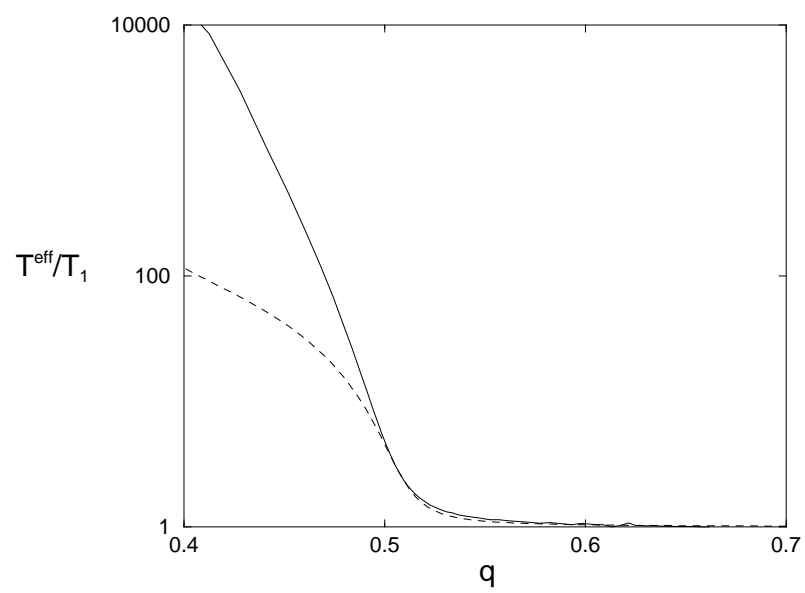

Fig. 2. Comparison of the effective and measured temperatures of the asymmetric spherical SK model for $T_{1}=10$, $J=20$ and $v=.1$. The solid line corresponds to the effective temperature of the bare model. The dashed line corresponds to the termometer measure.

If we consider a driven system with many times scales, the measured temperature over a certain time scale $q_{2}$ is the weighted average of all the effective temperatures of the system over this time scale. Since, on one hand, $R_{2}$ is a decreasing function of $q$ and, on the other hand, $T^{\mathrm{eff}}$ is an increasing function of $q$, we expect the measured temperature to be lower than the effective temperature when the thermometer probes the long time scales corresponding to the limit $q \rightarrow 0$. Nevertheless, for intermediate time scales, if $R_{2}\left(q, q_{2}\right)$ is not peaked sharply enough around $q_{2}$, it is possible to measure a temperature higher than the effective one, as shown, e.g., in figure 3 .

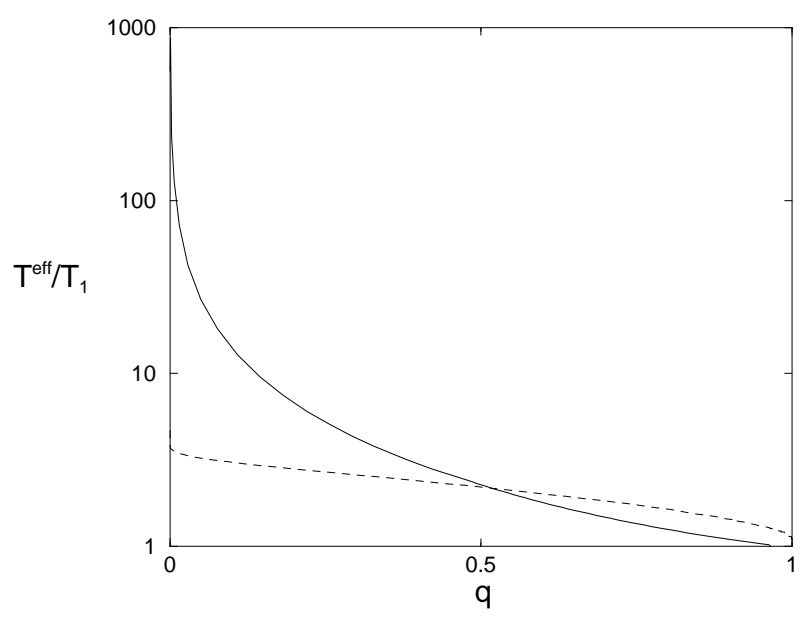

Fig. 3. Comparison of the effective and measured temperatures of the asymmetric spherical $\mathrm{SK}$ model for $T_{1}=10$, $J=20$ and $v=.9$. The solid line corresponds to the effective temperature of the bare model. The dashed line corresponds to the termometer measure.

\section{Effective temperature of a spherical SK model with randomly asymmetric bonds}

The previous considerations can be made more explicit in an exactly solvable model of a system-thermometer complex.

We consider a spherical SK model with randomly asymmetric bonds [10]. The Hamiltonian has the form

$$
H_{1}:=-\frac{1}{2} \sum_{i, j=1}^{N} J_{i j}^{\mathrm{s}} S_{1}^{i} S_{1}^{j}+\frac{r_{1}}{2} \sum_{i=1}^{N}\left(S_{1}^{i}\right)^{2} .
$$

The "spin" variables $S_{1}^{i}$ can take any real value. The parameter $r_{1}$ is a Lagrange multiplier which enforces the spherical constraint $\sum_{i=1}^{N}\left(S_{1}^{i}\right)^{2}=N$. The interaction matrix $J_{i j}^{\mathrm{s}}$ is a symmetric matrix whose diagonal elements vanish and whose off-diagonal elements, for each pair $\{i, j\}$ of indices, are independent Gaussian variables with zero mean and the following variance:

$$
\overline{\left(J_{i j}^{\mathrm{s}}\right)^{2}}=\frac{J^{2}}{N} \frac{1}{1+v^{2}} .
$$

The parameter $v>0$ is a measure of the strength of the driving force (see below). This spherical SK model is coupled to a single-spin paramagnetic thermometer via a bilinear interaction of strength $a$. The Hamiltonian of the 
paramagnet is given by

$$
H_{2}:=\frac{r_{2}}{2}\left(S_{2}\right)^{2}
$$

The response time scale of the paramagnet is given by $\tau_{2}:=1 / r_{2}$. Indeed, we recall that the bare response function of a paramagnet has the expression

$$
R_{2}(t)=\theta(t) \exp ^{-r_{2} t} .
$$

The total Hamiltonian writes

$$
H:=H_{1}\left(S_{1}\right)+H_{2}\left(S_{2}\right)+H_{\text {int }}\left(S_{1}, S_{2}\right),
$$

where the interaction Hamiltonian $H_{\text {int }}$ is given by

$$
H_{\mathrm{int}}:=-a S_{2} \sum_{i=1}^{N} S_{1}^{i}
$$

Stability requires $a<r_{1} r_{2}$. The dynamics of the system is described by a system of linear Langevin equations:

$$
\begin{aligned}
& \partial_{t} S_{1}^{i}(t)=-\frac{\partial H}{\partial S_{1}^{i}}+b_{i}\left(S_{1}\right)+\eta_{1}^{i}(t) \\
& \partial_{t} S_{2}(t)=-\frac{\partial H}{\partial S_{2}}+\eta_{2}(t) .
\end{aligned}
$$

In eq. (29), the driving field $b_{i}\left(S_{1}\right)$ is given by:

$$
b_{i}\left(S_{1}\right):=v J_{i j}^{\text {as }} S_{1}^{j}
$$

where $J_{i j}^{\text {as }}$ is an antisymmetric matrix whose off-diagonal elements, for each pair $\{i, j\}$ of indices, are independent Gaussian random variables of zero mean and variance equal to that of $J_{i j}^{\mathrm{s}}$ (eq. (24)). The $\eta_{1}^{i}$ are thermal noises at temperature $T_{1}$ with zero mean and variance given by $\left\langle\eta_{1}^{i}(t) \eta_{1}^{i}\left(t^{\prime}\right)\right\rangle=2 T_{1} \delta\left(t-t^{\prime}\right)$, while $\eta_{2}$ is a thermal noise at temperature $T_{2}$.

In the thermodynamical limit $(N \rightarrow \infty)$, it is possible to average over the disorder by the means of dynamical functional integration techniques. Thus the equations for the asymmetric spherical SK model in (29) reduce to a single equation for a single spin $S_{1}$. The new system of equations reads

$$
\begin{aligned}
\partial_{t} S_{1}(t)= & -r_{1}(t) S_{1}(t)+a S_{2}(t) \\
& +J^{\prime 2} \int_{t_{0}}^{t} d t^{\prime} R_{11}\left(t, t^{\prime}\right) S_{1}\left(t^{\prime}\right)+\eta_{1}(t) \\
\partial_{t} S_{2}(t)= & -r_{2} S_{2}(t)+a S_{1}(t)+\eta_{2}(t)
\end{aligned}
$$

where $J^{\prime}=\sqrt{\left(1-v^{2}\right) /\left(1+v^{2}\right)} J$, and $\eta_{1}$ is a renormalized Gaussian thermal noise with zero mean and variance given by

$$
\left\langle\eta_{1}(t) \eta_{1}\left(t^{\prime}\right)\right\rangle=2 T_{1} \delta\left(t-t^{\prime}\right)+J^{2} C_{11}\left(t, t^{\prime}\right) .
$$

For the response we obtain the following autonomous system:

$$
\left(\partial_{t}+r_{1}(t)\right) R_{11}\left(t, t^{\prime}\right)=a R_{21}\left(t, t^{\prime}\right)+\delta\left(t-t^{\prime}\right)
$$

$$
\begin{aligned}
& +J^{\prime 2} \int_{t_{0}}^{t} d t^{\prime \prime} R_{11}\left(t, t^{\prime \prime}\right) R_{11}\left(t^{\prime \prime}, t^{\prime}\right), \\
\left(\partial_{t}+r_{1}(t)\right) R_{12}\left(t, t^{\prime}\right)= & a R_{22}\left(t, t^{\prime}\right) \\
& +J^{\prime 2} \int_{t_{0}}^{t} d t^{\prime \prime} R_{11}\left(t, t^{\prime \prime}\right) R_{12}\left(t^{\prime \prime}, t^{\prime}\right), \\
\left(\partial_{t}+r_{2}\right) R_{22}\left(t, t^{\prime}\right)= & a R_{12}\left(t, t^{\prime}\right)+\delta\left(t-t^{\prime}\right), \\
\left(\partial_{t}+r_{2}\right) R_{21}\left(t, t^{\prime}\right)= & a R_{11}\left(t, t^{\prime}\right) .
\end{aligned}
$$

The time $t_{0}$ can be freely chosen between the time the interaction was switched on and the observation times $t$ and $t^{\prime}$. The equations for the correlation function involve the response:

$$
\begin{gathered}
\left(\partial_{t}+r_{1}\right) C_{11}\left(t, t^{\prime}\right)=a C_{21}\left(t, t^{\prime}\right)+2 T_{1} R_{11}\left(t^{\prime}, t\right) \\
+\int_{t_{0}}^{t} d t^{\prime \prime}\left(J^{\prime 2} R_{11}\left(t, t^{\prime \prime}\right) C_{11}\left(t^{\prime \prime}, t^{\prime}\right)\right. \\
\left.+J^{2} C_{11}\left(t, t^{\prime \prime}\right) R_{11}\left(t^{\prime}, t^{\prime \prime}\right)\right), \\
\left(\partial_{t}+r_{1}\right) C_{12}\left(t, t^{\prime}\right)=a C_{22}\left(t, t^{\prime}\right)+2 T_{1} R_{21}\left(t^{\prime}, t\right) \\
+\int_{t_{0}}^{t} d t^{\prime \prime}\left(J^{\prime 2} R_{11}\left(t, t^{\prime \prime}\right) C_{12}\left(t^{\prime \prime}, t^{\prime}\right)\right. \\
\left.+J^{2} C_{11}\left(t, t^{\prime \prime}\right) R_{21}\left(t^{\prime}, t^{\prime \prime}\right)\right), \\
\left(\partial_{t}+r_{2}\right) C_{22}\left(t, t^{\prime}\right)=a C_{12}\left(t, t^{\prime}\right)+2 T_{2} R_{22}\left(t^{\prime}, t\right), \\
\left(\partial_{t}+r_{2}\right) C_{21}\left(t, t^{\prime}\right)=a C_{11}\left(t, t^{\prime}\right)+2 T_{2} R_{12}\left(t^{\prime}, t\right) .
\end{gathered}
$$

After some time, the system enters a stationary regime where $C_{i j}\left(t, t^{\prime}\right)=\hat{C}_{i j}\left(t-t^{\prime}\right)$ and $R_{i j}\left(t, t^{\prime}\right)=\hat{R}_{i j}\left(t-t^{\prime}\right)$. Choosing $t_{0}, t^{\prime}$ and $t$ in this regime, and taking advantage of Fourier analysis, one can solve the system for the response and then the one for the correlation. For simplicity we suppose that the interaction between the system and the thermometer has been switched on an infinite time in the past. This corresponds to sending $t_{0} \rightarrow-\infty$. All the quantities depend on the value of $r_{1}$ which is chosen in order to verify the spherical condition: $C_{11}(t, t)=$ $\frac{1}{N} \sum_{i=1}^{N} S_{1}^{i^{2}}=1$. Taking the derivative of this condition with respect to time we obtain

$$
\lim _{t^{\prime} \rightarrow t^{-}} \frac{\partial C_{11}\left(t, t^{\prime}\right)}{\partial t}+\lim _{t^{\prime} \rightarrow t^{+}} \frac{\partial C_{11}\left(t, t^{\prime}\right)}{\partial t}=0 .
$$

Substituting eq. (39) we obtain an equation for $r_{1}$ :

$$
\begin{aligned}
r_{1}= & T+a C_{21}(0) \\
& +\left(J^{2}+J^{\prime 2}\right) \int_{t_{0}}^{t} d t^{\prime \prime} R_{11}\left(t, t^{\prime \prime}\right) C_{11}\left(t^{\prime \prime}, t^{\prime}\right) .
\end{aligned}
$$

Since $C_{21}, R_{11}$ and $C_{11}$ all depend on $r_{1}$ this is an equation for $r_{1}$. We solved it numerically and then substituted the value of $r_{1}$ into the correlation and response functions. After this step the solution is completed and we can search for the temperature $T_{2}$ of the paramagnetic thermometer which makes the heat flux to vanish.

The power exchanged between the thermometer and the system at stationarity is given by

$$
\dot{Q}_{2}:=\left\langle\frac{\partial H_{\mathrm{int}}}{\partial S_{1}} \dot{S}_{1}\right\rangle-\left\langle\frac{\partial H_{\mathrm{int}}}{\partial S_{2}} \dot{S}_{2}\right\rangle .
$$


From the definition (28) of $H_{\text {int }}$ we obtain

$$
\begin{aligned}
\dot{Q}_{2}(t) & =-a S_{2}(t) \frac{d S_{1}(t)}{d t}+a S_{1}(t) \frac{d S_{2}(t)}{d t} \\
& =\lim _{t^{\prime} \rightarrow t}\left(-a \partial_{t} C_{21}\left(t, t^{\prime}\right)+a \partial_{t} C_{12}\left(t, t^{\prime}\right)\right) .
\end{aligned}
$$

By using the results of the appendix and taking an inverse Fourier transformation, we finally obtain

$$
\begin{array}{r}
\dot{Q}_{2}=a \int \frac{d \omega}{2 \pi} i \omega\left(\tilde{C}_{12}(\omega)-\tilde{C}_{21}(\omega)\right) \\
=-2 a \int \frac{d \omega}{2 \pi} \omega \operatorname{Im}\left(\tilde{C}_{12}(\omega)\right)
\end{array}
$$

The temperature measured by the thermometer is the one which makes the heat flux $\dot{Q}_{2}$ to vanish. The resulting measured temperatures are shown in figure 5 and should be compared with the expected effective temperature shown in figure 4 .

\section{Results}

Figures 4 and 5 show the effective and measured values of the temperature as a function of the value of the correlation function, for the model under study. The two quantitites behave similarly, in that they are close to the equilibrium value $T_{1}$ for $q>q_{\mathrm{EA}}$, and start increasing, as $q$ becomes smaller and smaller, for $q<q_{\mathrm{EA}}$. Nevertheless it is possible to identify a quantitative discrepancy, analogous to the one discussed in section 2. For small values of the asymmetry parameter $v$, the driven system exhibits two clearly separated regimes, with $T^{\mathrm{eff}}=T_{1}$ for $q>q_{\mathrm{EA}}$, and a temperature increase for $q<q_{\mathrm{EA}}$. For high values of $q, T^{\text {meas }}$ remains close to $T_{1}$, but is much smaller than $T^{\mathrm{eff}}$ in the low- $q$ region, as shown in fig. 2. For higher $v, T^{\mathrm{eff}}$ increases smoothly all along the range of $q$. Being an average of $T^{\mathrm{eff}}$ over a given time scale, $T^{\text {meas }}$ becomes quickly higher than $T_{1}$ as it feels the increasing of $T^{\mathrm{eff}}$ even for short time scales. Then it keeps on increasing, at a slower pace than $T^{\text {eff }}$, as shown in fig. 3. Figures 6 show the ratio $T^{\text {meas }} / T^{\text {eff }}$ as a function of the asymmetry parameter $v$. The ratio remains close to the ideal value 1 only for $q>q_{\mathrm{EA}}$. Ideally, similar plots would apply to an aging system as a function of the inverse waiting time, because of the correspondence between $v$ and the waiting time discussed above.

In figure 7 and 8 the behavior of the measured and of the effective temperature is shown as a function of the asymmetry parameter $v$ is shown for different values of the thermometer response time $\tau_{2}$. The figures are in qualitative agreement only for short time scales which are represented by the lower lines of the plots. When the thermometer probes longer times scales, $T^{\text {meas }}\left(\tau_{2}\right)$ shows a non monotonic behaviour which does not appear in the $T^{\mathrm{eff}}(\tau)$ plot. In the waiting time representation, a thermometer with a fixed (but long) reaction time $\tau_{2}$ would first yield higher and higher readings, as it attempts to approach the flat part of the graph shown in fig. 1, but

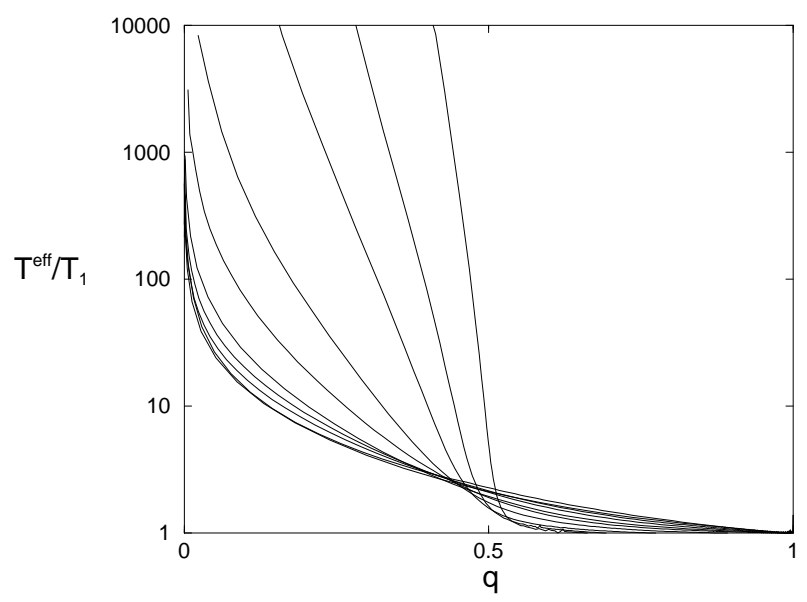

Fig. 4. Effective temperature $T^{\mathrm{eff}}$ vs. $q$ for the bare asymmetric spherical SK model for $T_{1}=10, J=20$, and for different values of the asymmetry parameter $v . T^{\mathrm{eff}}$ is normalized by the temperature $T_{1}$ of the thermal bath coupled to the aging system. The lines correspond (from above to below) to $v=0.1 ; 0.15 ; 0.2 ; 0.3 ; 0.4 ; 0.5 ; 0.6 ; 0.7 ; 0.8 ; 0.9$. The EdwardsAnderson order parameter $q_{E A}$ of the corresponding symmetric model is given by $q_{E A}=T_{1} / J=.5$, and corresponds to the value of $q$ from where the curves diverge.

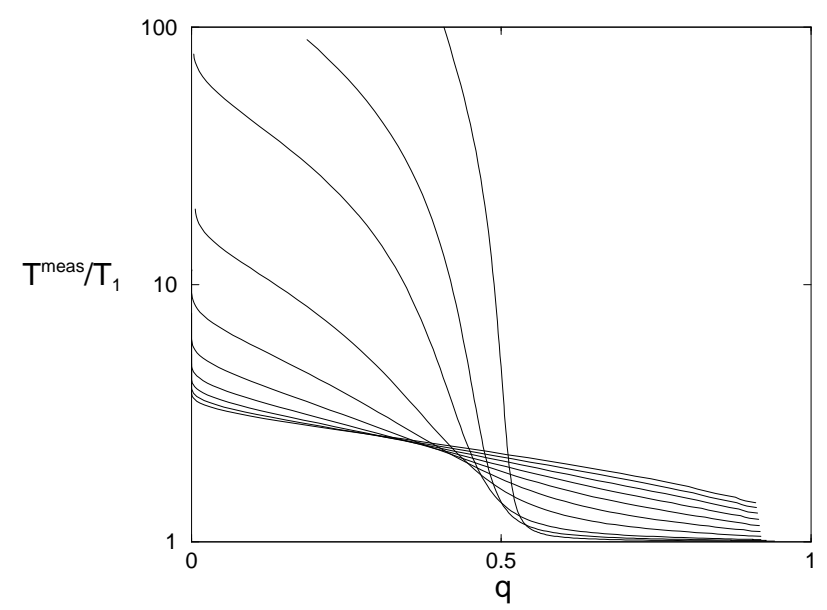

Fig. 5. Measured effective temperature $T^{\text {meas }}$ vs. $q$ of the asymmetric spherical SK model for $T_{1}=10, J=$ $20, a=.1$, and for different values of the asymmetry $v$. The lines correspond (from above to below) to $v=$ $0.1 ; 0.15 ; 0.2 ; 0.3 ; 0.4 ; 0.5 ; 0.6 ; 0.7 ; 0.8 ; 0.9$.

will eventually read the temperature of the thermal bath as $t_{\mathrm{w}} \gg \tau_{2}$. This leads to a non monotonic behavior as a function of $t_{\mathrm{w}}$, and an analogous one as a function of $v$, at least for sufficiently slow thermometers.

In conclusion we have shown in an exactly solvable model of a thermometric measurement in a "glassy" system that, while thermometer do indeed measure temperatures higher than the one of the environment if they are slow enough, the relation of the measured temperature 
with the effective temperature defined, e.g., in [4] is far from trivial.

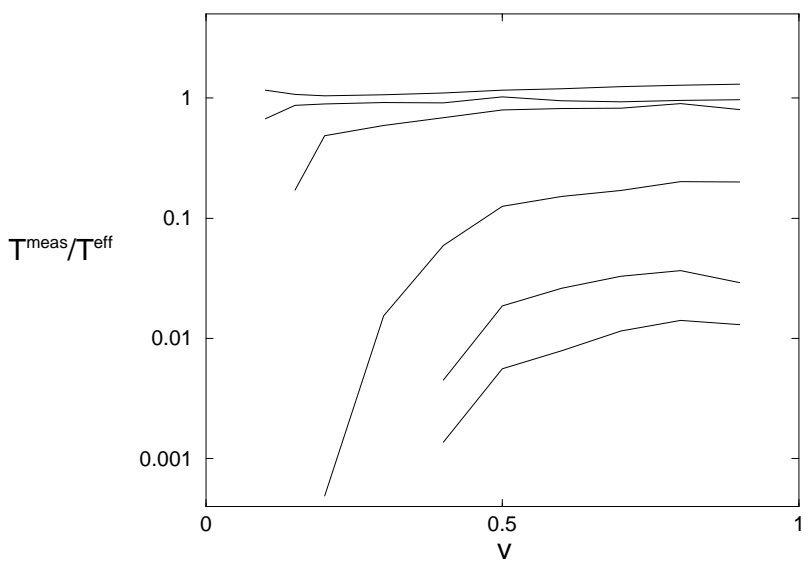

Fig. 6. Ratio $T^{\text {meas }} / T^{\mathrm{eff}} v s$. asymmetry parameter $v$ of the asymmetric spherical SK model for $T_{1}=10, J=20$, and for different values of the overlap $q$. The lines correspond (from above to below) to $q=0.8 ; 0.5 ; 0.4 ; 0.1 ; 0.01 ; 0.001$.

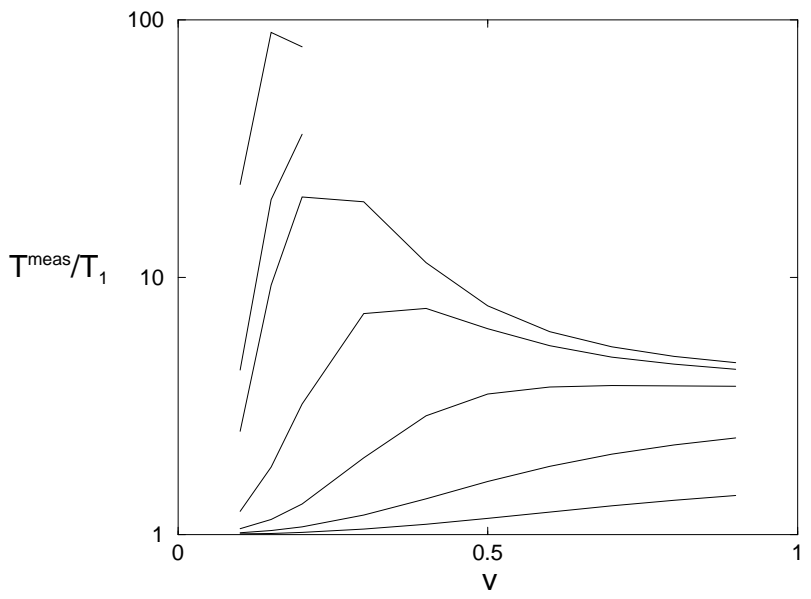

Fig. 7. Measured effective temperature $T^{\text {meas }}$ vs. asymmetry parameter $v$ of the asymmetric spherical SK model for $T_{1}=10$, $J=20, a=.1$, and for different values of the parameter $\tau_{2}$ which sets the characteristic time of the thermometer. $T^{\text {meas }}$ is normalized by the temperature $T_{1}$ of the thermal bath coupled to the aging system. The lines correspond (from above to below) to $\tau_{2}=100 ; 10 ; 1.0 ; 0.1 ; 0.01$.

LP acknowledges the support of a Chaire Joliot of the ESPCI. Discussions with Serge Galam are gratefully acknowledged.

\section{References}

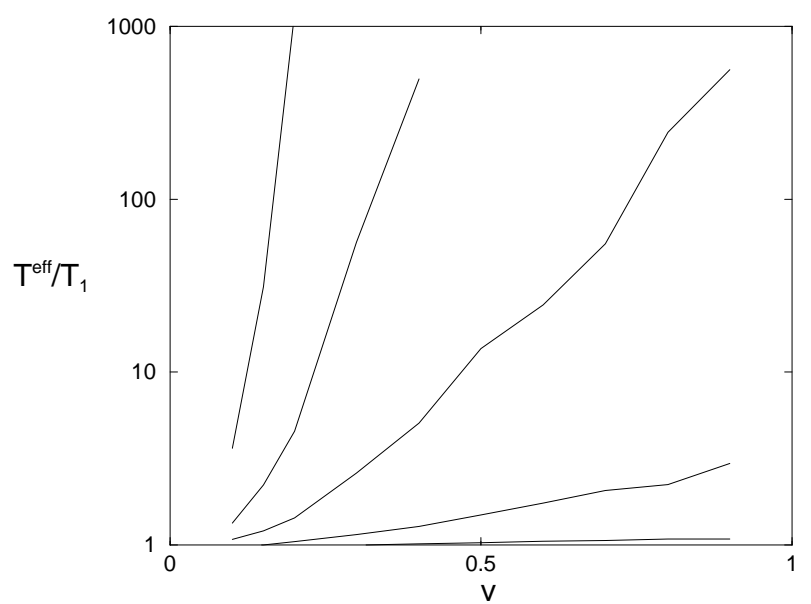

Fig. 8. Effective temperature $T^{\mathrm{eff}} v s$. asymmetry parameter $v$ of the asymmetric spherical SK model for $T_{1}=10, J=20$ and for different values of $\tau$. T $T^{\text {eff }}$ is normalized by the temperature $T_{1}$ of the thermal bath coupled to the aging system. The lines correspond (from above to below) to $\tau=100 ; 10 . ; 1 . ; 0.1 ; 0.01$.

1. S.-K. Ma, Statistical Mechanics (World Scientific, Singapore 1985), p. 3.

2. P. C. Hohenberg and B. I. Shraiman, Physica 37 (1989) 109.

3. L. F. Cugliandolo and J. Kurchan, J. Phys. A27 (1994) 5749 .

4. L. F. Cugliandolo, J. Kurchan and L. Peliti, Phys. Rev. E55 (1997) 3898.

5. L. F. Cugliandolo and J. Kurchan, Physica A263 (1999) 242.

6. L. F. Cugliandolo, cond-mat/9903250.

7. N. E. Israeloff and T. S. Grigera, Europhys. Lett. 43 (1998) 308; T. Grigera and N. E. Israeloff, cond-mat/9904351.

8. H. Horner, Z. Phys. B57 (1984) 29; ibid B57 (1984) 39.

9. R. Exartier and L. Peliti, to be published in Physics Letters A.

10. A. Crisanti and H. Sompolinsky, Phys. Rev A36 (1987) 4922.

\section{Appendix}

Taking the Fourier transform of the autonomous system for response functions, equations (35 38), we obtain

$$
\begin{aligned}
& \left(i \omega+r_{1}\right) \tilde{R}_{11}(\omega)=a \tilde{R}_{21}(\omega)+J^{\prime 2} \tilde{R}_{11}(\omega)^{2}+1 \\
& \left(i \omega+r_{1}\right) \tilde{R}_{12}(\omega)=a \tilde{R}_{22}(\omega)+J^{\prime 2} \tilde{R}_{11}(\omega) \tilde{R}_{12}(\omega) \\
& \left(i \omega+r_{2}\right) \tilde{R}_{22}(\omega)=a \tilde{R}_{12}(\omega)+1 \\
& \left(i \omega+r_{2}\right) \tilde{R}_{21}(\omega)=a \tilde{R}_{11}(\omega)
\end{aligned}
$$

Again, from (39 42) we obtain the following equation for the correlation functions:

$$
\begin{aligned}
\left(i \omega+r_{1}\right) \tilde{C}_{11}(\omega)= & a \tilde{C}_{21}(\omega)+J^{\prime 2} \tilde{R}_{11}(\omega) \tilde{C}_{11}(\omega) \\
& +J^{2} \tilde{C}_{11}(\omega) \overline{\tilde{R}_{11}(\omega)}+2 T_{1} \overline{\tilde{R}_{11}(\omega)} \\
\left(i \omega+r_{1}\right) \tilde{C}_{12}(\omega)= & a \tilde{C}_{22}(\omega)+J^{\prime 2} \tilde{R}_{11}(\omega) \tilde{C}_{12}(\omega)
\end{aligned}
$$




$$
\begin{aligned}
& +J^{2} \tilde{C}_{11}(\omega) \overline{\tilde{R}_{21}(\omega)}+2 T_{1} \overline{\tilde{R}_{21}(\omega)} \\
\left(i \omega+r_{2}\right) \tilde{C}_{22}(\omega)= & a \tilde{C}_{12}(\omega)+2 T_{2} \overline{\tilde{R}_{22}(\omega)} \\
\left(i \omega+r_{2}\right) \tilde{C}_{21}(\omega)= & a \tilde{C}_{11}(\omega)+2 T_{2} \overline{\tilde{R}_{12}(\omega)}
\end{aligned}
$$

In Fourier space, the equation (44) for the spherical parameter $r_{1}$ writes:

$$
\begin{aligned}
r_{1}(t)= & T+a \int \frac{d \omega}{2 \pi} \tilde{C}_{21}(\omega) \\
& +\left(J^{2}+J^{\prime 2}\right) \int \frac{d \omega}{2 \pi} \tilde{R}_{11}(\omega) \tilde{C}_{11}(\omega) .
\end{aligned}
$$

Equations 48 56 form a nine-equation system for the response and correlation functions and for the spherical parameter, which is possible to solve explicitly. Equations (48) and (49) yield a second-order algebraic equation for $R_{11}(\omega)$ :

$$
J^{\prime 2} \tilde{R}_{11}^{2}-\left(i \omega+r_{1}-\frac{a^{2}}{i \omega+r_{2}}\right) \tilde{R}_{11}+1=0
$$

We choose the solution which respects the symmetries of $\operatorname{Im}\left(\tilde{R}_{11}(\omega)\right), \operatorname{Re}\left(\tilde{R}_{11}(\omega)\right)$, and recovers the right value for $\tilde{R}_{11}(\omega)$ in the limit $a \rightarrow 0$.

From equations (49) and (50) we obtain

$$
\tilde{R}_{12}(\omega)=\tilde{R}_{21}(\omega)=\frac{a}{i \omega+r_{2}} \tilde{R}_{11}(\omega),
$$

while equation (50) yields:

$$
\tilde{R}_{22}(\omega)=\frac{1}{i \omega+r_{2}}+\frac{a^{2}}{\left(i \omega+r_{2}\right)^{2}} \tilde{R}_{11}(\omega) .
$$

From equations (52) and (55) we obtain:

$$
\begin{aligned}
\tilde{C}_{11} & =\frac{2 T_{1}+2 T_{2} a^{2} /\left(\omega^{2}+r_{2}^{2}\right)}{\left|\tilde{R}_{11}\right|^{-2}-J^{2}}, \\
\tilde{C}_{21} & =\frac{a}{\omega^{2}+r_{2}^{2}}\left[\left(r_{2}-i \omega\right) \tilde{C}_{11}+2 T_{2} \overline{\tilde{R}_{11}(\omega)}\right] .
\end{aligned}
$$

As shown by equation (47), the measurement procedure of $T^{\text {meas }}$ gives a particular relevance to the imaginary part of $\tilde{C}_{21}(\omega)$ :

$$
\operatorname{Im}\left(\tilde{C}_{21}\right)=-a \frac{2 T_{2} \operatorname{Im}\left(\tilde{R}_{11}\right)+\omega \tilde{C}_{11}}{\omega^{2}+r_{2}^{2}}
$$

